\title{
IDENTIFICATION OF POTENTIAL SALMONELLA TYPHI BETA-LACTAMASE TEM 1 INHIBITORS USING PEPTIDOMIMETICS, VIRTUAL SCREENING, AND MOLECULAR DYNAMICS SIMULATIONS
}

\author{
RAKESH K. R. PANDIT1, DINESH GUPTA2, TAPAN K. MUKHERJEE3* ${ }^{*}$ \\ 1Department of Biotechnology, Chandigarh Group of Colleges, Landran, Mohali, Punjab, ${ }^{2}$ Translational Bioinformatics Group, \\ International Centre of Genetic Engineering and Biotechnology, New Delhi, ${ }^{1,3}$ Department of Biotechnology, Maharishi Markandeshwar \\ University, Mullana, Ambala \\ Email: tapanmu@yahoo.com
}

Received: 21 Jul 2017 Revised and Accepted: 22 Nov 2017

\section{ABSTRACT}

Objective: The purpose of this study was to identify a potential peptidomimetic $S$. typhi Beta-lactamase TEM 1 inhibitor to tackle the antibiotic resistance among S. typhi.

Methods: The potential peptidomimetic inhibitor was identified by in silico docking of the small peptide WFRKQLKW with S. typhi Beta-lactamase TEM 1. The 3D coordinate geometry of the residues of small peptide interacting with the active site of the receptor was generated and mimics were identified using PEP: MMs: MIMIC server. All the identified mimics were docked at the active site of the receptor using Autodock 4.2 and the bestdocked complex was selected on the basis of binding energy and number of H-bonds. The complex was then subjected to molecular dynamics simulations of $30 \mathrm{~ns}$ using AMBER 12 software package. The stereochemical stability of the Beta-lactamase TEM 1-WFRKQLKW complex was estimated with the help of Ramachandran plot using PROCHECK tool.

Results: In the present study, a new potential peptidomimetic inhibitor (ZINC05839264) of Beta-lactamase TEM 1 has been identified based on antimicrobial peptide WFRKQLKW by virtual screening of the MMsINC database. The docking and molecular simulation studies revealed that the mimic binds more tightly to the active site of the receptor than the peptide. The Ramachandran plot also shows that the Beta-lactamase TEM 1mimic complex is stereo chemically more stable than Beta-lactamase TEM 1-WFRKQLKW complex as more number of residues (93.6\%) are falling under the core region of the plot in case of the former.

Conclusion: The study shows that the peptidomimetic compound can act as a potential inhibitor of S. typhi Beta-lactamase TEM 1 and further it can be developed into more effective therapeutic to tackle the problem of antibiotic resistance.

Keywords: S. typhi, Beta-lactamase TEM 1, Antibiotic Resistance, Virtual screening, Docking, Peptidomimetics, Molecular dynamics simulation

(C) 2018 The Authors. Published by Innovare Academic Sciences Pvt Ltd. This is an open access article under the CC BY license (http://creativecommons.org/licenses/by/4.0/) DOI: http://dx.doi.org/10.22159/ijpps.2018v10i1.21520

\section{INTRODUCTION}

Salmonella typhi is a facultative anaerob, rod-shaped, flagellated gram-negative bacteria belonging to Enterobacteriaceae family [1] that causes Salmonellosis in humans. Salmonellosis can be identified in three different clinical forms [2] Gastroenteritis, Septicemia, Enteric fevers. Most Salmonella infections fall under gastroenteritis diseases. The common signs and symptoms of the infection include nausea, vomiting, abdominal cramps, diarrhea, fever, chills, headache, and blood in the stool. The symptoms of the infection by Salmonella normally last for four to seven days, while it commonly takes several months for the bowel to become normal. Typhoid fever is generally caused by only a few strains of Salmonella, which is considered to be a common and deadly disease in some of the developing countries. S. typhi is found to be endemic to developing countries such as Central and South America, Africa, and the Indian subcontinent. According to a study in developing countries, 33 million cases each year has been estimated, which is lower in developed countries like the UK and the USA as the majority of cases include travelers returning from endemic areas [3].

Multi-Drug Resistance (MDR) among Salmonella has become a global concern which has been proved to be a major obstacle in disease management. Lack of knowledge, efficient regulatory provisions [4] and easy access to antibiotics has been additive factors that have elevated the problem, especially in developing countries. According to the Centers for Disease Control and Prevention (CDC), there have been $3800 S$. typhi infection cases in the US every year, out of which $67 \%$ of the cases are drug-resistant. The number of infectious cases in India is twice as that of the US [5] which is due to easy access, socio-economic growth, and absence of regulatory provisions. The increasing cases of antibiotic resistance have led the scientific community to focus on the development of the alternative therapeutics to combat the problem. Beta-lactamase TEM 1 has been found to be one of the main factors which provide resistance to antibiotics in pathogenic bacteria by breaking the beta-lactam ring of the penicillin class of antibiotics [6]. This has generated an urge to the development of alternative therapeutics with higher efficacy and low toxicity.

Antimicrobial peptides (AMPs) are short, low molecular weight, broad-spectrum proteins with sizes ranging from as short as 2 amino acid residues in the case of synthetic peptides to around 100 amino acid residues in the case of naturally occurring peptides. AMPs have been found to be involved in providing an innate immune response to host against pathogenic bacteria, viruses and fungi [7]. Numerous studies have been conducted on the development of AMPs as an alternative to the antibiotics as major pathogenic bacteria have developed antibiotic resistance.

Due to broad-spectrum activity and different mode of action, AMPs can be seen as an alternative to various antibiotics. Until May 2016, more than 2500 naturally occurring antimicrobial peptides have already been reported on Antimicrobial Peptide Database (http://aps.unmc.edu/AP/main.php). Despite their distinguished mode of action, AMPs are not considered as the go-to approach for developing therapeutic agents due to their non-specificity, toxicity, poor stability, poor absorption, the high cost of production, degradation by proteases, and development of allergies by the individuals for peptides [8]. 
Peptidomimetics has evolved as a great tool for designing and developing drugs with greater pharmacodynamic and pharmacokinetic properties. Peptidomimetic compounds mimic the essential elements of the AMPs by retaining their antimicrobial activity but are also more stable hence are more bioavailable, possess specificity and are less susceptible to degradation by proteases [9]. Peptidomimetics are designed by identifying the key residues involved in the biological activity through SAR model. The structural constraints of the essential residues are used to further screen the compound libraries (virtual screening). The mimics can be further screened by docking them with the receptor and analyzing their binding energies. The current study aims at the identification of new potential drug candidates against $S$. typhi Beta-lactamase TEM 1 [10] based on the peptidomimetics, virtual screening, and molecular dynamics simulation.

\section{MATERIALS AND METHODS}

\section{Methods}

Identification and three-dimensional modeling of small peptide

The small peptide WFRKQLKW [11] was identified and the threedimensional structure of the selected peptide was predicted with PEPstr Mod server [12, 13].

\section{Docking studies and identification of active residues in peptide}

The docking of the peptide was performed using Clusproproteinprotein docking server [14-18] with antibody mode which resulted in a number of models out of which the best model was selected based on the highest cluster size. The residues of the peptide involved in interaction with the active site of the receptor were identified using DeLano Scientific PyMol [19].

\section{Virtual screening}

Various peptidomimetic compounds were identified via virtual screening against the peptide-receptor complex using PEPMMsMIMIC [20] web tool for the virtual screening of peptide mimetic compounds.

\section{Docking and interaction studies of peptidomimetic compounds}

All the screened peptidomimetic compounds were docked to the receptor using Autodock4.2 [21] docking tool and the hydrogen bonding interactions of the compounds with the receptor active site were identified and analyzed using DeLano Scientific PyMol.

\section{Screening of the potential drug candidate}

The potential drug candidate was selected on the basis of drug likeliness parameters such as Lipinski's Rule of five [22], Ghose filter [23] and Blood-Brain Barrier likeliness filter using DruLiTo tool (http://www.niper.gov.in/pi_dev_tools/DruLiToWebl).

\section{Molecular Dynamics and Simulation}

The most potential peptidomimetic drug candidate in complex with the receptor was further subjected to molecular dynamics and simulation with AMBER12 [24] software using ff99SB [25] force field and TIP3P water model [26].

\section{RESULTS AND DISCUSSION}

The 3D structure of the peptide was predicted using PEPstrMOD server (fig. 1).

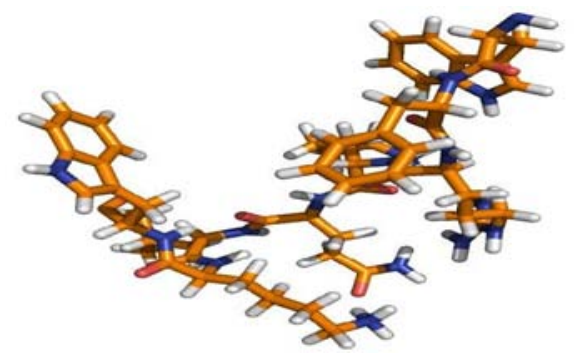

Fig. 1: Predicted 3D structure of the peptide WFRKQLKW

The docking studies of the peptide WFRKQLKW showed the close binding of the antimicrobial peptide in the active site cavity of the Beta-lactamase TEM 1 (fig. 4A, a). Based on the interacting residues of the antimicrobial peptide with that of the active site residues of the receptor, virtual screening of the small compounds was performed using PEPMMsMIMIC server which search peptidomimetics compounds based on 3-points pharmacophore and USR amino acid annotation parameters of the interacting residues of the peptide with the active site of the receptor from the MMsINC database which contain 3,967,056 entries.

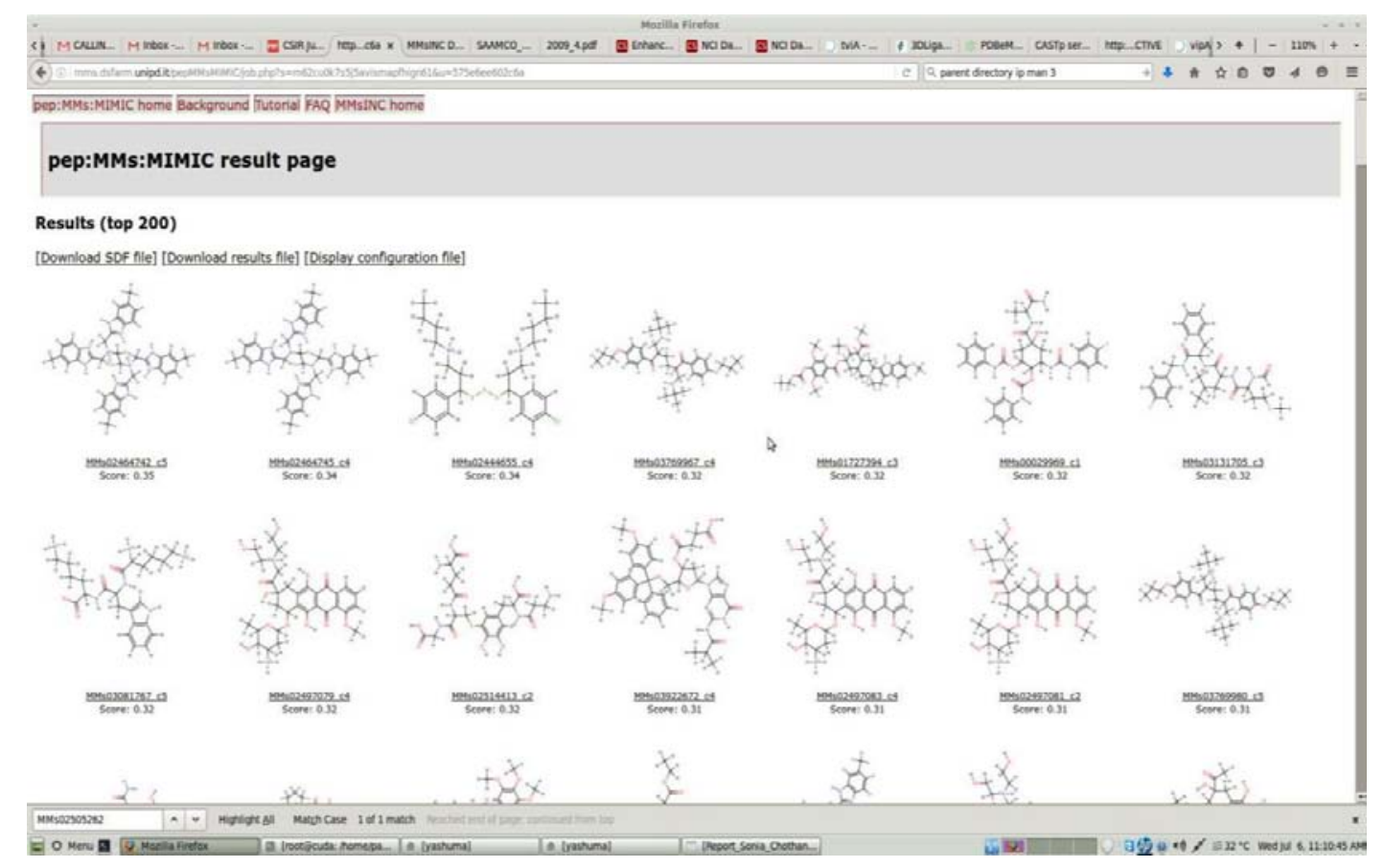

Fig. 2: Various peptidomimetic compounds searched by virtual screening based on the 3-points pharmacophore and USR amino acid notation parameters of the interacting residues of the peptide with the active site of $S$. typhi Beta-lactamase TEM 1 
The virtual screening by PEPMMsMIMIC resulted in 200 peptidomimetic compounds (fig. 2). The docking parameters for all the screened mimics were generated using raccoon [27] which is a graphical interface for preparing ligands for performing AutoDock virtual screenings. All the mimics were then docked against the active site of $S$. typhi Beta-lactamase TEM-1 using AutoDock which generated a number of poses for each mimic. The best-docked compound (ZINCID: ZINC05839264, fig. 3) was selected on the basis of the drug likeliness parameters such as Lipinski's Rule of five, Ghose filter and Blood-Brain Barrier likeliness. The interaction of the peptidomimetic compound with the active site of the receptor is shown in fig. $4 \mathrm{~B}, \mathrm{~b}$ and table 1.

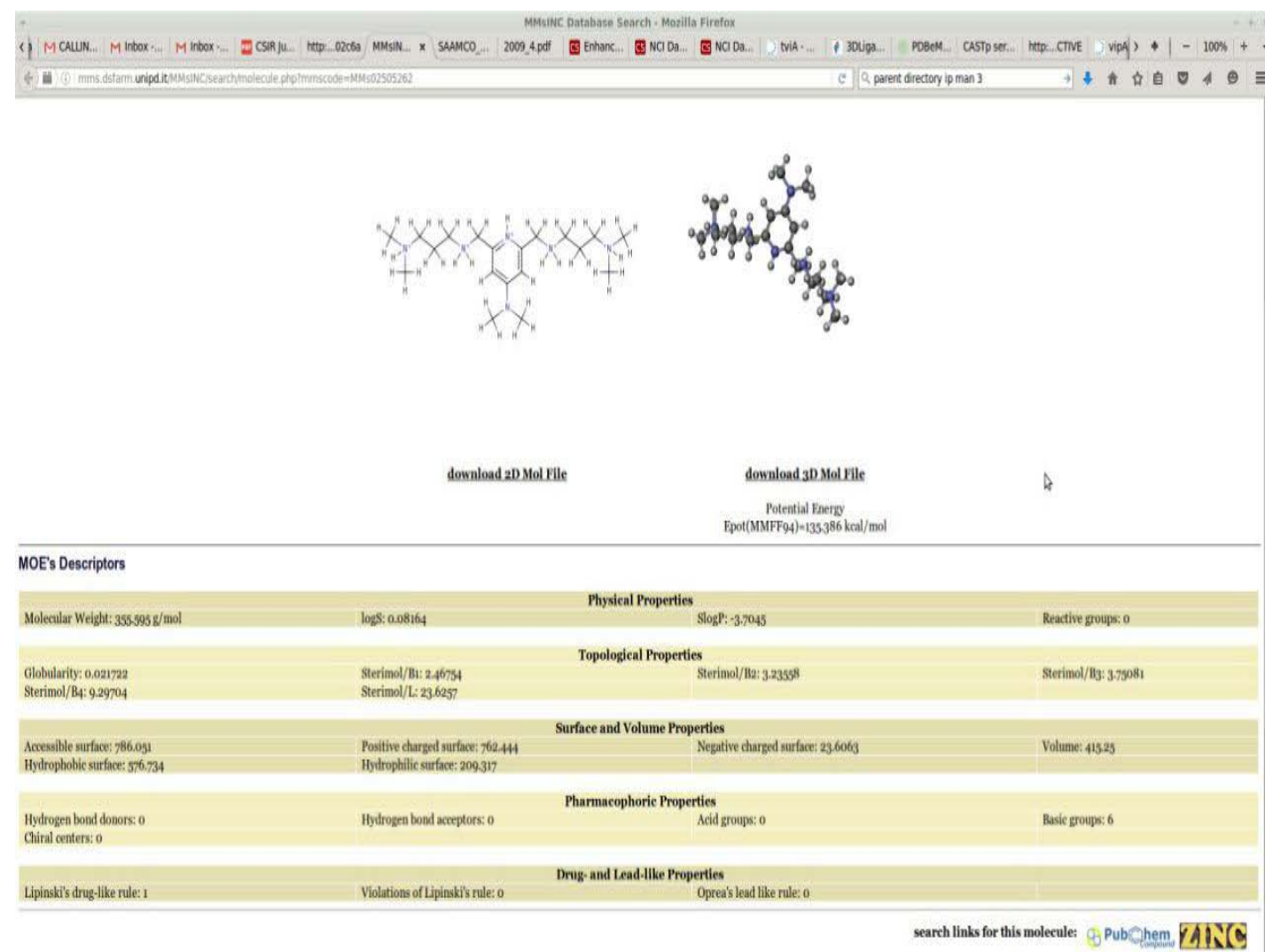

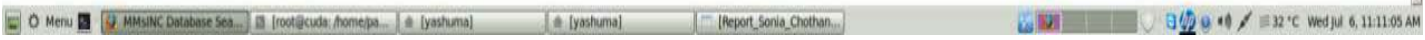

Fig. 3: Virtual screened peptidomimetic showing the 3D structure and various drug-like properties
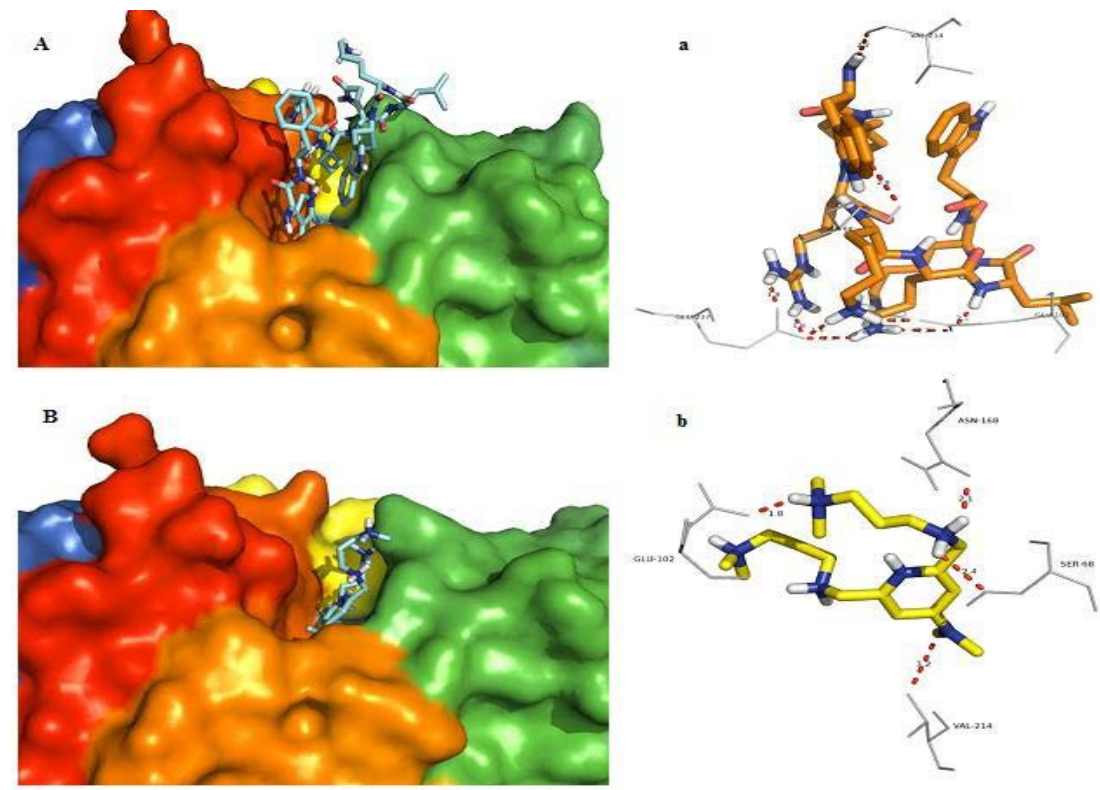

Fig. 4: In silico docking. Surface view representing a binding mode of antimicrobial peptide (A) and peptidomimetic compound (B) at the active site of Beta-lactamase TEM 1. Non-covalent interactions of the antimicrobial peptide (a) and peptidomimetic compound (b) with the active site residues of Beta-lactamase TEM 1 
Table 1: Various non-bonded interactions between receptor active site and Antimicrobial peptide and peptidomimetic compound (ZINC05839264)

\begin{tabular}{lll}
\hline Receptor-ligand interactions & & Peptidomimetic \\
\hline Receptor residue name & Antimicrobial peptide & 3.2 \\
\cline { 2 - 3 } & H-bond distance(Å) & 2.4 \\
VAL214 & 2.2 & 1.8 \\
GLU102 & 1.9 & 2.9 \\
\hline
\end{tabular}

Both the complexes were subjected to molecular dynamics simulations. Energy minimization (fig. 5A, a), temperature (fig. 5B, b) and density (fig. 5C, c) equilibration revealed that both the systems were equilibrated at very similar values of energy, density, and temperature. The production MD revealed that the peptide complex showed consistent root mean square deviation (RMSD) whereas the peptidomimetic complex firstly showed a steep increase in RMSD and then stabilized after 20 ns simulation (fig. 5 D, d). Both systems were observed to be stable after $20 \mathrm{~ns}$ in a $30 \mathrm{~ns}$ simulation run.
The stereo-chemical stability of both the complexes was studied with Ramachandran plot generated using PROCHECK [28] tool at Swiss-model [29]. The Ramachandran plot (fig. 6) for both complexes showed that more than $90 \%$ residues were falling under the core regions of the plot which reveals that both the complexes were stereo-chemically stable. The plot also revealed that the Betalactamase TEM 1-Peptidomimetic complex was more stereochemically stable than Beta-lactamase TEM 1-Peptide complex as 93.6\% residues were in the core region in the former as compared $92.2 \%$ residues in the latter.
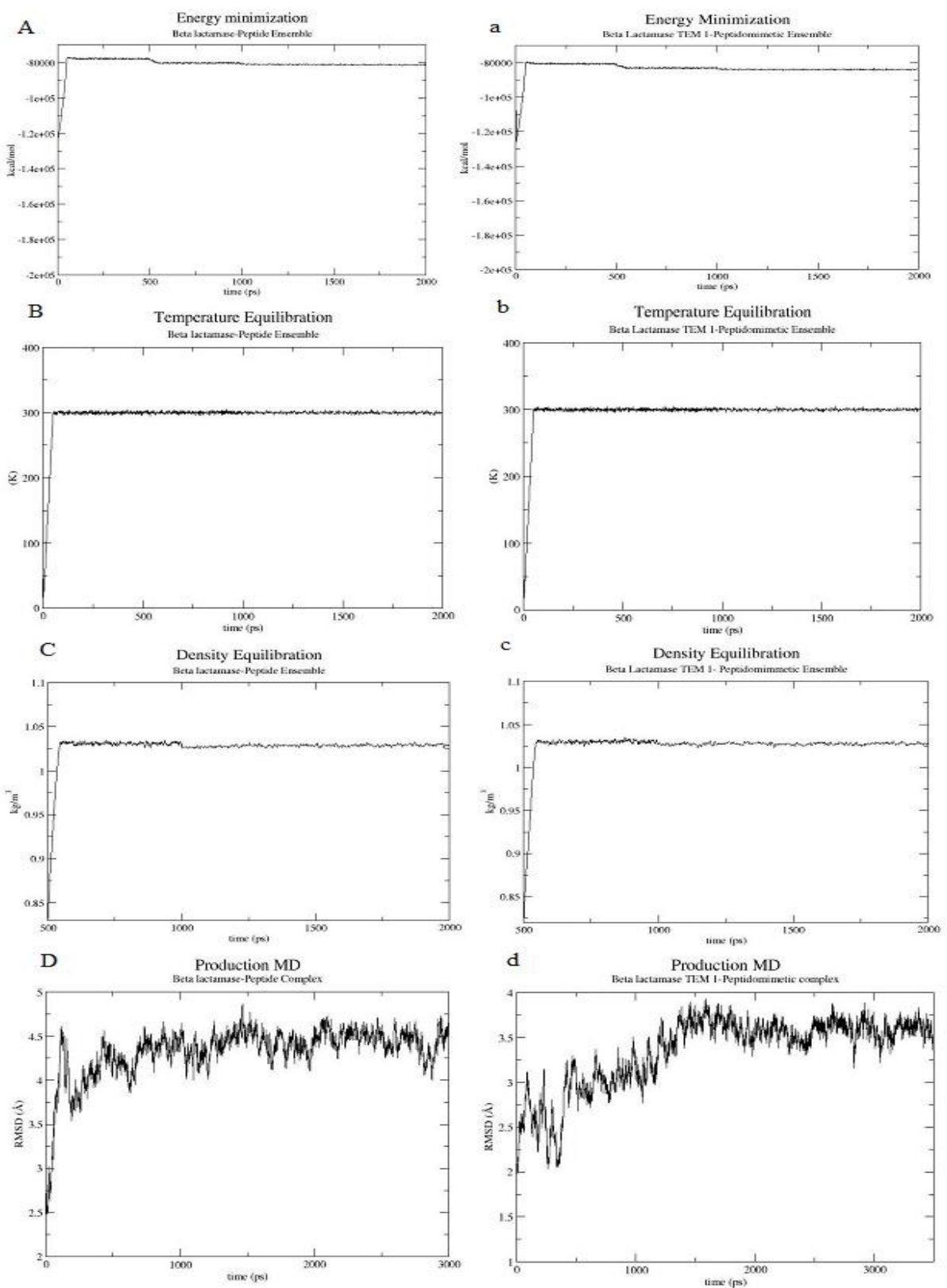

Fig. 5: Molecular dynamics and simulation results of the receptor-peptide complex (A, B, C, D) and receptor-peptidomimetic complex (a, b, c, d) showing close similarity in both the cases over the entire course of the simulation 

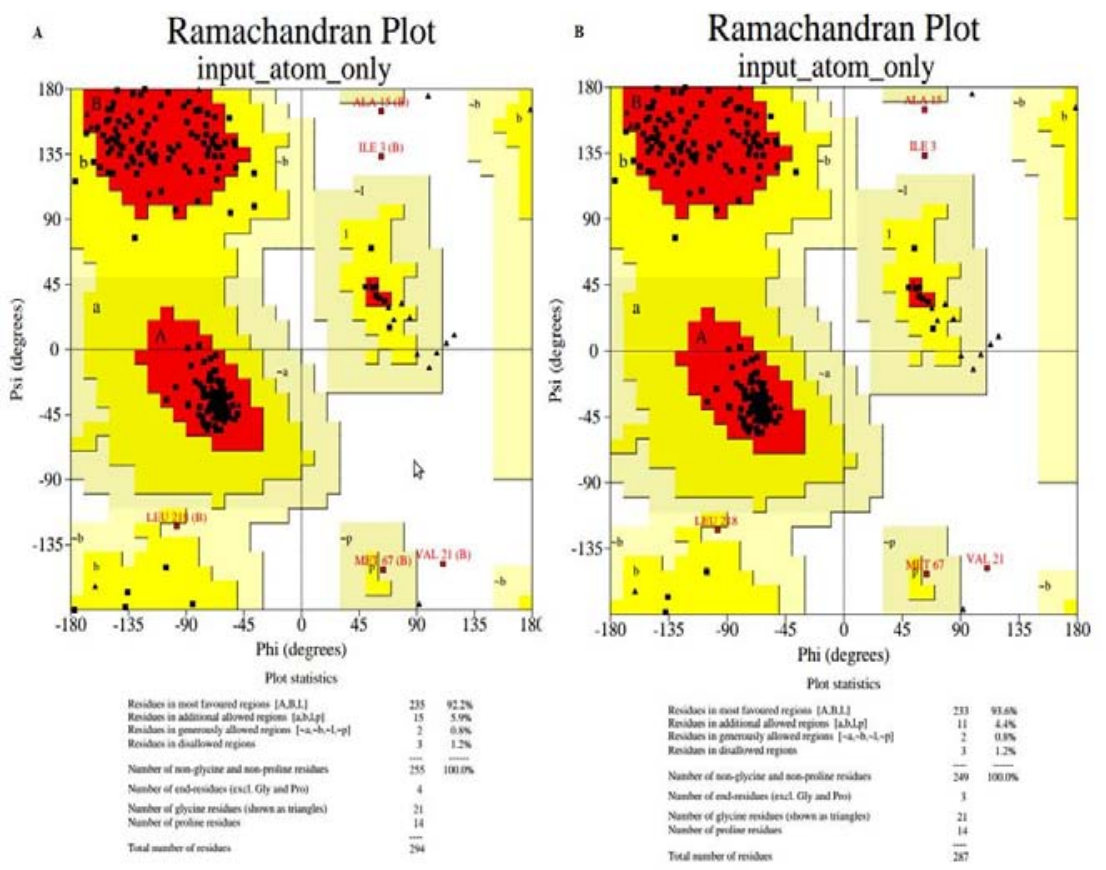

Fig. 6: Ramachandran plots of beta lactamase-peptide complex (A) and Beta lactamase-peptidomimetic complex (B) showing stereochemical stability as more than $\mathbf{9 0 \%}$ residues are falling under the core region of the plot

\section{CONCLUSION}

In this study, we have identified ZINC05839264 as a potential $S$. typhi Beta-lactamase TEM1 inhibitor through virtual screening based on the interaction of the compound with the active site of the receptor. The docking studies, production MD revealed that the mimic compound binds more closely to the active site cavity than the antimicrobial peptide which indicates the better efficiency of the compound as compared to the antimicrobial peptide. The Ramachandran plot analysis showed that the Beta lactamasePeptidomimetic complex was more stable than Beta lactamasePeptide complex as more number of residues i.e. $93.6 \%$ was falling under the core region of the plot in the former than $92.2 \%$ of the latter. It is concluded from the study that the peptidomimetic compound can be developed in a potential inhibitor of $S$. typhi Betalactamase TEM 1. It is suggested that further modifications in mimic's structure may be studied to enhance its antimicrobial activity. The antimicrobial activity of the mimics may be checked via in vitro studies so that a validated activity of the compound can be established.

\section{AUTHORS CONTRIBUTIONS}

The work was carried out by Rakesh $\mathrm{Kr}$ Pandit under the supervision of Dr. Dinesh Gupta. The manuscript was checked and revised by Dr. Tapan K Mukherjee.

\section{CONFLICT OF INTERESTS}

The author declares no conflict of interest

\section{REFERENCES}

1. Andino A, Hanning I. Salmonella enterica: survival, colonization, and virulence differences among serovars. Sci World J 2015:1-16. http://dx.doi.org/10.1155/2015/520179.

2. Gray, Jeffrey T, Paula J, Fedorka-Cray. Salmonella. Foodborne Diseases. Eds. Dean O. Cliver and Hans P. Riemann. San Francisco: Academic Press; 2002. p. 55-68.

3. John Threlfall E. Antimicrobial drug resistance in salmonella: problems and perspectives in food-and water-borne diseases. FEMS Microbiol Rev 2002;26:141-8.

4. Laxminarayan R, Chaudhury RR. Antibiotic resistance in India: drivers and opportunities for action. PLoS Med 2016;13:e1001974.
5. Thomas P, Boeckel V, Brower C, Gilbert M, Grenfell BT, Levin $\mathrm{SA}$, et al. Global trends in antimicrobial use in food animals. PNAS 2015; 112:5649-54.

6. Livermore DM. $\beta$-Lactamases in laboratory and clinical resistance. Clin Microbiol Rev 1995;8:557-84.

7. Izadpanah A, Gallo RL. Antimicrobial peptides. J Am Acad Dermatol 2005;52:381-90.

8. Bradshaw J. Cationic antimicrobial peptides: issues for potential clinical use. Biol Drugs 2003;17:233-40.

9. Vagner J, Qu H, H ruby VJ. Peptidomimetics, a synthetic tool of drug discovery. Curr Opin Chem Biol 2008;12:292-6.

10. Pandit RK, Mukherjee TK, Kumar A, Kumar V, Kaur PS. Structure prediction and assessment of beta-lactamase TEM-1 from S. typhi using molecular dynamics and simulation studies. Int J Rec Sci Res 2016;7:9509-13.

11. Mishra B, Leishangthem GD, Gill K, Singh AK, Das S, Singh K, et $a l$. A novel antimicrobial peptide derived from modified $\mathrm{N}$ terminal domain of bovine lactoferrin: Design, synthesis, activity against multidrug-resistant bacteria and Candida. Biochim Biophysica Acta 2013;1828:677-86.

12. Singh S, Singh H, Tuknait A, Chaudhary K, Singh B, Kumaran S, et al. PEPstr MOD: structure prediction of peptides containing natural, non-natural and modified residues. Biol Direct 2015;10:73.

13. Kaur H, Garg A, Raghava GPS. PEPstr: a de novo method for tertiary structure prediction of small bioactive peptides. Protein Pept Lett 2007;14:626-30.

14. Kozakov D, Beglov D, Bohnuud T, Mottarella S, Xia B, Hall DR, et al. How good is automated protein docking? Proteins: Struct Funct Bioinf 2013;81:2159-66.

15. Kozakov D, Brenke R, Comeau SR, Vajda S. PIPER: an FFT-based protein docking program with pairwise potentials. Proteins 2006;65:392-406.

16. Comeau SR, Gatchell DW, Vajda S, Camacho CJ. ClusPro: an automated docking and discrimination method for the prediction of protein complexes. Bioinformatics 2004;20:45-50.

17. Comeau SR, Gatchell DW, Vajda S, Camacho CJ. ClusPro: a fully automated algorithm for protein-protein docking. Nucleic Acids Res 2004;32:W96-9.

18. Brenke R, Hall DR, Chuang GY, Comeau SR, Bohnuud T, Beglov $\mathrm{D}$, et al. Application of asymmetric statistical potentials to antibody-protein docking. Bioinformatics 2012;28:2608-14.

19. The PyMOL Molecular Graphics System, Version 1.8 Schrödinger, LLC; 2015. 
20. Floris M, Masciocchi J, Fanton M, Moro S. Swimming into peptidomimetic chemical space using pepMMsMIMIC. Nucl Acids Res 2011;39 Suppl 2:W261-W269.

21. Morris GM, Huey R, Lindstrom W, Sanner MF, Belew RK, Goodsell D, et al. Autodock4 and AutoDockTools4: automated docking with selective receptor flexibility. J Comp Chem 2009;16:2785-91.

22. Lipinski CA. Lead-and drug-like compounds: the rule-of-five revolution. Drug Discovery Today Technol 2004;1:337-41.

23. Ghose AK, Viswanadhan VN, Wendoloski JJ. A knowledge-based approach in designing combinatorial or medicinal chemistry libraries for drug discovery. 1. A qualitative and quantitative characterization of known drug databases. J Comb Chem 1999;1:55-68.

24. Case DA, Darden TA, Cheatham TE III, Simmerling CA, Wang J, Duke RE, et al. AMBER 12, University of California, San Francisco; 2012.
25. Lindorff-Larsen K, Piana S, Palmo K, Maragakis P, Klepeis JL, Dror RO, et al. Improved side-chain torsion potentials for the Amber ff99SB protein force field. Proteins 2010;78:1950-8.

26. Jorgensen WL, Chandrasekhar J, Madura JD, Impey RW, Klein Comparison of simple potential functions for simulating liquid water. J Chem Phys 1983;79:926-35.

27. Forli S, Huey R, Pique ME, Sanner MF, Goodsell DS, Olson AJ. Computational protein-ligand docking and virtual drug screening with the Auto Dock suite. Nat Protocols 2016;11:905-19.

28. Laskowski RA, MacArthur MW, Moss D, Thornton JM. PROCHECK: a program to check the stereochemical quality of protein structures. J Appl Cryst 1993;26:283-91.

29. Arnold K, Bordoli L, Kopp J, Schwede T. The SWISS-MODEL workspace: a web-based environment for protein structure homology modelling. Bioinformatics 2006;22:195-201. 